\title{
ON FOCAL SUBMANIFOLDS OF ISOPARAMETRIC HYPERSURFACES AND SIMONS FORMULA
}

\author{
QICHAO LI AND LI ZHANG ${ }^{\dagger}$
}

\begin{abstract}
The focal submanifolds of isoparametric hypersurfaces in spheres are all minimal Willmore submanifolds, mostly being $\mathcal{A}$-manifolds in the sense of A.Gray but rarely Ricci-parallel ( QTY, [LY], [TY3]). In this paper we study the geometry of the focal submanifolds via Simons formula. We show that all the focal submanifolds with $g \geq 3$ are not normally flat by estimating the normal scalar curvatures. Moreover, we give a complete classification of the semiparallel submanifolds among the focal submanifolds.
\end{abstract}

\section{INTRODUCTION}

Isoparametric hypersurfaces in a unit sphere are hypersurfaces with constant principal curvatures. They consist of a one-parameter family of parallel hypersurfaces which laminates the unit sphere with two focal submanifolds at the end. It is remarkable that the focal submanifolds of isoparametric hypersurfaces provide infinitely many spherical submanifolds with abundant intrinsic and extrinsic geometric properties. For instance, they are all minimal Willmore submanifolds in unit spheres and mostly $\mathcal{A}$-manifolds in the sense of A.Gray ([Gra]), except for two cases of $g=6$ (cf. QTY], [TY2], [LY], [Xie]). As is well known, an Einstein manifold minimally immersed in a unit sphere is Willmore, however the focal submanifolds are rarely Ricci-parallel, thus rarely Einstein (cf. [TY3]).

In this paper we study the geometry of the focal submanifolds in terms of Simons formula (see $[\mathrm{Sim}],[\mathrm{CdK}]$ ). To state our results clearly, we firstly give some preliminaries on isoparametric hypersurfaces and their focal submanifolds in unit spheres.

A remarkable result of Münzner ([Mün] states that the number $g$ of distinct principal curvatures must be 1,2,3,4 or 6 by the arguments of differential topology. Moreover, the isoparametric hypersurfaces can be represented as the regular level sets in a unit sphere of some Cartan-Münzner polynomial F. By a Cartan-Münzner polynomial, we

2000 Mathematics Subject Classification. 53A30, 53C42.

Key words and phrases. isoparametric hypersurface, focal submanifold, semiparallel, normally flat. $\dagger$ The second author is the corresponding author. 
mean a homogeneous polynomial $F$ of degree $g$ on $\mathbb{R}^{n+2}$ satisfying the so-called CartanMünzner equations:

$$
\left\{\begin{array}{l}
|\nabla F|^{2}=g^{2} r^{2 g-2}, \quad r=|x|, \\
\Delta F=c r^{g-2}, \quad c=g^{2}\left(m_{2}-m_{1}\right) / 2,
\end{array}\right.
$$

where $\nabla F$ and $\Delta F$ are the gradient and Laplacian of $F$ on $\mathbb{R}^{n+2}$. The function $f=\left.F\right|_{\mathbb{S}^{n+1}}$ takes values in $[-1,1]$. The level sets $f^{-1}(t)(-1<t<1)$ gives the isoparametric hypersurfaces. In fact, if we order the principal curvatures $\lambda_{1}>\cdots>\lambda_{g}$ with multiplicities $m_{1}, \cdots, m_{g}$, then $m_{i}=m_{i+2}(\bmod g)$, in particular, all multiplicities are equal when $g$ is odd. On the other hand, the critical sets $M_{+}:=f^{-1}(1)$ and $M_{-}:=f^{-1}(-1)$ are connected submanifolds with codimensions $m_{1}+1$ and $m_{2}+1$ in $\mathbb{S}^{n+1}$, called focal submanifolds of this isoparametric family.

The isoparametric hypersurfaces $M^{n}$ with $g \leq 3$ in $\mathbb{S}^{n+1}$ were classified by Cartan to be homogeneous ([Car1], Car2]). For $g=6$, Abresch ([Abr] $)$ proved that $m_{1}=m_{2}=$ 1 or 2. Dorfmeister-Neher ([DN]) and Miyaoka (Miy3 ) showed they are homogeneous, respectively. For $g=4$, all the isoparametric hypersurfaces are recently proved to be OT-FKM type $([\mathrm{OT}],[\mathrm{FKM}])$ or homogeneous with $\left(m_{1}, m_{2}\right)=(2,2),(4,5)$, except possibly for the unclassified case with $\left(m_{1}, m_{2}\right)=(7,8)$ or $(8,7)$ ([CCJ], [Imm, [Chi]).

Recently, Tang and Yan ([TY3]) provided a complete determination for which focal submanifolds of $g=4$ are Ricci-parallel except for the unclassified case $\left(m_{1}, m_{2}\right)=$ $(7,8)$ or $(8,7)$. More precisely, they proved

Theorem 1.1. ([TY3]) For the focal submanifolds of isoparametric hypersurfaces in spheres with $g=4$, we have

(i) The $M_{+}$of OT-FKM type is Ricci parallel if and only if $\left(m_{1}, m_{2}\right)=(2,1),(6,1)$, or it is diffeomorphic to $S p(2)$ in the homogeneous case with $\left(m_{1}, m_{2}\right)=(4,3)$; While the $M_{-}$of OT-FKM type is Ricci-parallel if and only if $\left(m_{1}, m_{2}\right)=$ $(1, k)$.

(ii) For $\left(m_{1}, m_{2}\right)=(2,2)$, the one diffeomorphic to $\widetilde{G}_{2}\left(\mathbb{R}^{5}\right)$ is Ricci-parallel, while the other diffeomorphic to $\mathbb{C} P^{3}$ is not.

(iii) For $\left(m_{1}, m_{2}\right)=(4,5)$, both are not Ricci-parallel.

Inspired by Tang and Yan's result, we give a complete classification of the semiparallel submanifolds among all the focal submanifolds via Simons formula.

Recall that semiparallel submanifolds were introduced by Deprez in 1986 (see Dep), as a generalization of parallel submanifolds. Given an isometric immersion $f: M \rightarrow N$, denote by $B$ and $\bar{\nabla}$ its second fundamental form and the connection in (tangent bundle) $\oplus$ (normal bundle), respectively, the immersion is said to be semiparallel if

$$
(\bar{R}(X, Y) \cdot B)(Z, W):=\left(\bar{\nabla}_{X Y}^{2} B\right)(Z, W)-\left(\bar{\nabla}_{Y X}^{2} B\right)(Z, W)=0
$$


for any tangent vectors $X, Y, Z$ and $W$ to $M$, where $\bar{R}$ is the curvature tensor of the connection $\bar{\nabla}$ in (tangent bundle) $\oplus$ (normal bundle). An elegant survey on the study of semiparallel submanifolds in a real space form can be found in Lum.

Our main results state as follows:

Theorem 1.2. For focal submanifolds of isoparametric hypersurfaces in unit spheres with $g \geq 3$, we have

(i) For $g=3$ : all the focal submanifolds are semiparallel.

(ii) For $g=4$ : the $M_{+}$of OT-FKM type with $\left(m_{1}, m_{2}\right)=(2,1),(6,1)$, the $M_{+}$of homogeneous OT-FKM type with $\left(m_{1}, m_{2}\right)=(4,3)$, all of the $M_{-}$of OT-FKM type with $\left(m_{1}, m_{2}\right)=(1, k)$ and the focal submanifold diffeomorphic to $\widetilde{G}_{2}\left(\mathbb{R}^{5}\right)$ with $\left(m_{1}, m_{2}\right)=(2,2)$ are semiparallel. None of the others are semiparallel.

(iii) For $g=6$ : none of the focal submanifolds are semiparallel.

Remark 1.1. Our results work on the unclassified case of $g=4,\left(m_{1}, m_{2}\right)=(7,8)$ or $(8,7)$.

This paper is organized as follows. In Section 2, we firstly state some preliminaries on geometry of submanifolds and estimate the normal scalar curvatures of focal submanifolds. In the next section, we proved Theorem 1.2 via Simons formula .

\section{Notations And Preliminaries}

Let $f: M^{n} \rightarrow \mathbb{S}^{n+p}$ be a compact $n$-dimensional minimal submanifold of the unit sphere. Denote by $B$ the second fundamental form, $\bar{\nabla}$ the covariant derivative with respect to the connection in (tangent bundle) $\oplus$ (normal bundle), $\nabla\left(\nabla^{\perp}\right)$ the induced Levi-Civita (normal) connection in the tangent (normal) bundle, respectively. Choosing a local field $\xi_{1}, \cdots, \xi_{p}$ of orthonormal frames of $T^{\perp} M$, we set $A_{\alpha}=A_{\xi_{\alpha}}$, the shape operator with respect to $\xi_{\alpha}$.

The first covariant derivative of $B$ is defined by

$$
\left(\bar{\nabla}_{X} B\right)(Z, W)=(\bar{\nabla} B)(X, Z, W):=\nabla_{X}^{\perp} B(Z, W)-B\left(\nabla_{X} Z, W\right)-B\left(Z, \nabla_{X} W\right) .
$$

By the Codazzi equation, $\left(\bar{\nabla}_{X} B\right)(Z, W)$ is symmetric in the tangent vectors $X, Z$ and $W$.

Define the second covariant derivative of $B$ by

$$
\left(\bar{\nabla}_{X Y}^{2} B\right)(Z, W):=\left(\bar{\nabla}_{X}(\bar{\nabla} B)\right)(Y, Z, W)=(\bar{\nabla}(\bar{\nabla} B))(X, Y, Z, W),
$$

where $X, Y, Z, W \in T M$. And the (rough) Laplacian of $B$ is defined by

$$
(\Delta B)(X, Y):=\sum_{i=1}^{n}\left(\bar{\nabla}_{e_{i} e_{i}}^{2} B\right)(X, Y),
$$


where $e_{1}, \cdots, e_{n}$ is a local orthonormal frame of $T M$.

The famous Simons formula states as follows:

Lemma 2.1. ([Sim $[\overline{\mathrm{CdK}}]$ ) Denote by $\|B\|^{2}$ the squared norm of the 2 nd fundamental form of a submanifold $M^{n}$ minimally immersed in unit sphere $\mathbb{S}^{n+p}$, then

$$
\begin{aligned}
\frac{1}{2} \Delta\|B\|^{2} & =\|\bar{\nabla} B\|^{2}+\langle B, \Delta B\rangle \\
& =\|\bar{\nabla} B\|^{2}+n\|B\|^{2}-\sum_{\alpha, \beta=1}^{p}\left\langle A_{\alpha}, A_{\beta}\right\rangle^{2}-\sum_{\alpha, \beta=1}^{p}\left\|\left[A_{\alpha}, A_{\beta}\right]\right\|^{2},
\end{aligned}
$$

where $\left\langle A_{\alpha}, A_{\beta}\right\rangle:=\operatorname{tr}\left(A_{\alpha} A_{\beta}\right),\left\|\left[A_{\alpha}, A_{\beta}\right]\right\|^{2}:=-\operatorname{tr}\left(A_{\alpha} A_{\beta}-A_{\beta} A_{\alpha}\right)^{2}$ and $\Delta:=\operatorname{tr}\left(\nabla^{2}\right)$, the Laplace operator of $M^{n}$.

The scalar curvature of the normal bundle is defined as $\rho^{\perp}=\left\|R^{\perp}\right\|$, where $R^{\perp}$ is the curvature tensor of the normal bundle. By the Ricci equation $\left\langle R^{\perp}(X, Y) \xi, \eta\right\rangle=$ $\left\langle\left[A_{\xi}, A_{\eta}\right] X, Y\right\rangle$, the normal scalar curvature can be rewritten as

$$
\rho^{\perp}=\sqrt{\sum_{\alpha, \beta=1}^{p}\left\|\left[A_{\alpha}, A_{\beta}\right]\right\|^{2}} .
$$

Clearly, the normal scalar curvature is always nonnegative and the $M^{n}$ is normally flat if and only if $\rho^{\perp}=0$, and by a result of Cartan, which is equivalent to the simultaneous diagonalisation of all shape operators $A_{\xi}$.

Lemma 2.2. ([CR $]$ The focal submanifolds of isoparametric hypersurfaces are austere submanifolds in unit spheres, and the shape operators of focal submanifolds are isospectral, whose principal curvatures are $\cot \left(\frac{k \pi}{g}\right), 1 \leq k \leq g-1$ with multiplicities $m_{1}$ or $m_{2}$, alternately.

Proposition 2.1. For focal submanifolds of isoparametric hypersurfaces in $\mathbb{S}^{n+1}$ with $g \geq 3$, the squared norms of the second fundamental forms $\|B\|^{2}$ are constants:

(i) For the cases of $g=3$ and multiplicities $m_{1}=m_{2}:=m$,

$$
\|B\|_{M_{ \pm}}^{2}=\frac{2}{3} m(m+1), \quad m=1,2,4,8 .
$$

(ii) For the cases of $g=4$ and multiplicities $\left(m_{1}, m_{2}\right)$,

$$
\|B\|_{M_{+}}^{2}=2 m_{2}\left(m_{1}+1\right) \text {. }
$$

Similarly,

$$
\|B\|_{M_{-}}^{2}=2 m_{1}\left(m_{2}+1\right) .
$$

(iii) For the cases of $g=6$,

$$
\|B\|_{M_{ \pm}}^{2}=\frac{20}{3} m(m+1), \quad m=1,2 .
$$


Proof. The proof follows easily from $\|B\|^{2}=\sum_{\alpha=1}^{p} \operatorname{tr}\left(A_{\alpha}^{2}\right)$ and Lemma 2.2.

Theorem 2.1. For focal submanifolds of isoparametric hypersurfaces in $\mathbb{S}^{n+1}$ with $g \geq 3$, the normal scalar curvatures are estimated as follows:

(i) For the cases of $g=3$ and multiplicities $m_{1}=m_{2}=: m$,

$$
\rho_{M_{ \pm}}^{\perp}=\frac{2}{3} m \sqrt{2(m+1)}, \quad m \in\{1,2,4,8\} .
$$

(ii) For the cases of $g=4$ and multiplicities $\left(m_{1}, m_{2}\right)$,

$$
\rho_{M_{+}}^{\perp} \geq \sqrt{2 m_{1} m_{2}\left(m_{1}+1\right)}, \quad \rho_{M_{-}}^{\perp} \geq \sqrt{2 m_{1} m_{2}\left(m_{2}+1\right)} .
$$

(iii) For the cases of $g=6$ and multiplicities $m_{1}=m_{2}=: m$,

$$
\left(\rho_{M_{+}}^{\perp}\right)^{2}=\left(72+\frac{8}{9}\right) m^{2}(m+1), \quad\left(\rho_{M_{-}}^{\perp}\right)^{2}=\frac{80}{9} m^{2}(m+1) \quad m \in\{1,2\} .
$$

As a result, all the focal submanifolds with $g \geq 3$ are not normally flat.

Proof. By definition, the normal scalar curvature is given by

$$
\begin{aligned}
\left(\rho^{\perp}\right)^{2} & =\sum_{\alpha, \beta=1}^{p}\left\|\left[A_{\alpha}, A_{\beta}\right]\right\|^{2}=\sum_{\alpha, \beta=1}^{p} \operatorname{tr}\left(A_{\alpha} A_{\beta}-A_{\beta} A_{\alpha}\right)\left(A_{\beta} A_{\alpha}-A_{\alpha} A_{\beta}\right) \\
& =2 \operatorname{tr} \sum_{\alpha, \beta=1}^{p}\left(A_{\alpha}^{2} A_{\beta}^{2}\right)-2 \operatorname{tr} \sum_{\alpha, \beta=1}^{p}\left(A_{\alpha} A_{\beta}\right)^{2} .
\end{aligned}
$$

We estimate the normal scalar curvatures case by case.

(i) For the cases of $g=3$ and multiplicities $m_{1}=m_{2}=: m, n=2 m, p=m+1$. Let $\xi$ be an arbitrary unit normal vector of a focal submanifold, by Lemma 2.2 , the shape operator $A_{\xi}$ has two distinct eigenvalues, $\frac{1}{\sqrt{3}}$ and $-\frac{1}{\sqrt{3}}$, with the same multiplicities $m$. It follows easily that

$$
\left(A_{\xi}\right)^{2}=\frac{1}{3} \mathrm{Id} .
$$

Since the shape operators defined on the unit normal bundle are isospectral, for any two orthogonal unit normal vectors $\xi$ and $\eta, A_{\frac{1}{\sqrt{2}}(\xi+\eta)}$ still satisfies

$$
A_{\frac{1}{\sqrt{2}}(\xi+\eta)}^{2}=\frac{1}{3} \mathrm{Id}
$$

that is

$$
\frac{1}{2} A_{\xi}^{2}+\frac{1}{2} A_{\xi} A_{\eta}+\frac{1}{2} A_{\eta} A_{\xi}+\frac{1}{2} A_{\eta}^{2}=\frac{1}{3} \mathrm{Id},
$$

hence

$$
A_{\xi} A_{\eta}+A_{\eta} A_{\xi}=0
$$

which follows

$$
\operatorname{tr}\left(A_{\xi} A_{\eta}\right)^{2}=-\operatorname{tr}\left(A_{\eta}^{2} A_{\xi}^{2}\right)=-\operatorname{tr}\left(A_{\xi}^{2} A_{\eta}^{2}\right) .
$$


Substituting (5) and (6) into (44), one has

$$
\left(\rho_{M_{ \pm}}^{\perp}\right)^{2}=8 \sum_{1 \leq \alpha<\beta \leq p} \operatorname{tr}\left(A_{\alpha}^{2} A_{\beta}^{2}\right)=8 \cdot \frac{2 m}{9} \cdot \frac{m(m+1)}{2}=\frac{8 m^{2}(m+1)}{9} .
$$

(ii) For the cases of $g=4$ and multiplicities $\left(m_{1}, m_{2}\right)$ : Recall a formula of OzekiTakeuchi (see $[\mathrm{OT}]$ p.534 Lemma. 12. (ii))

$$
A_{\alpha}=A_{\beta}^{2} A_{\alpha}+A_{\alpha} A_{\beta}^{2}+A_{\beta} A_{\alpha} A_{\beta}, \quad \alpha \neq \beta .
$$

Multiply by $A_{\beta}$ on both sides,

$$
A_{\alpha} A_{\beta}=A_{\beta}^{2} A_{\alpha} A_{\beta}+A_{\alpha} A_{\beta}^{3}+A_{\beta} A_{\alpha} A_{\beta}^{2}, \quad \alpha \neq \beta .
$$

Taking traces of both sides and observing that $A_{\alpha}^{3}=A_{\alpha}$ for a focal submanifold with $g=4$, we arrive at that

$$
\left\langle A_{\alpha}, A_{\beta}\right\rangle:=\operatorname{tr}\left(A_{\alpha} A_{\beta}\right)=0, \quad \alpha \neq \beta .
$$

On the other hand, for a focal submanifold with $g=4$, says $M_{+},\|B\|^{2}=$ $2 m_{2}\left(m_{1}+1\right)$ (by Proposition 2.1), $\left\langle A_{\alpha}, A_{\alpha}\right\rangle=2 m_{2}$ (by Lemma 2.2). Substituting these into Simons' formula (2), we conclude that

$$
\begin{aligned}
\sum_{\alpha, \beta=1}^{p} \|\left.\left[A_{\alpha}, A_{\beta}\right]\right|_{M_{+}} ^{2} & =\|\bar{\nabla} B\|^{2}+2 m_{2}\left(m_{1}+1\right)\left(2 m_{2}+m_{1}\right)-4 m_{2}^{2}\left(m_{1}+1\right) \\
& =\|\bar{\nabla} B\|^{2}+2 m_{1} m_{2}\left(m_{1}+1\right) \geq 2 m_{1} m_{2}\left(m_{1}+1\right) .
\end{aligned}
$$

The calculations for $M_{-}$are completely similarly.

(iii) For the cases of $g=6$ and multiplicities $m_{1}=m_{2}=: m$, all the focal submanifolds are homogeneous, and the result follows from a straightforward calculation and the explicit expressions for $A_{\alpha}$ 's from Miyaoka (Miy1 Miy2]).

\section{Semiparallel submanifolds and Simons formula}

For a semiparallel submanifold $M^{n}$ immersed in $\mathbb{S}^{n+p}$, denote by $e_{1}, \cdots, e_{n}$ a local orthonormal frame of $T M, X, Y \in T M$, and $H$ the mean curvature vector of $M^{n}$, then

$$
\begin{aligned}
(\Delta B)(X, Y) & :=\sum_{i=1}^{n}\left(\bar{\nabla}_{e_{i} e_{i}}^{2} B\right)(X, Y)=\sum_{i=1}^{n}\left(\bar{\nabla}_{X Y}^{2} B\right)\left(e_{i}, e_{i}\right) \\
& =\bar{\nabla}_{X Y}^{2}\left(\sum_{i=1}^{n} B\left(e_{i}, e_{i}\right)\right)=\bar{\nabla}_{X Y}^{2}(H)=0,
\end{aligned}
$$

where the second equality follows from the definition of semiparallel submanifolds and Codazzi equation, and the third from the commutativity of the covariant differentiations and taking trace. By the arbitrariness of $X, Y \in T M$, one concludes that $\Delta B=0$ for a minimal semiparallel submanifold. 
On the other hand, for a focal submanifold of an isoparametric hypersurface, $\|B\|^{2}=$ const. (by Proposition 2.1), $H=0$ (by Lemma 2.2), combining with the Simons formula $\frac{1}{2} \Delta\|B\|^{2}=\|\bar{\nabla} B\|^{2}+\langle B, \Delta B\rangle$ one arrives at that $\|\bar{\nabla} B\|^{2}+\langle B, \Delta B\rangle=0$, thus a focal submanifold of an isoparametric hypersurface is semiparallel if and only if $\|\bar{\nabla} B\|^{2}=0$, namely, it is a parallel submanifold. It remains to give a complete classification of the focal submanifolds satisfying $\|\bar{\nabla} B\|^{2}=0$.

It is convenient to define the covariant derivative $\left(\bar{\nabla}_{X} A\right)_{\alpha}$ of $B$ along $\xi_{\alpha} \in U^{\perp} M$ by $\left\langle\left(\bar{\nabla}_{X} A\right)_{\alpha} Y, Z\right\rangle=\left\langle(\bar{\nabla} B)(X, Y, Z), \xi_{\alpha}\right\rangle$. It follows easily that

$$
\left(\bar{\nabla}_{X} A\right)_{\alpha} Y=\left(\nabla_{X} A_{\alpha}\right) Y-\sum_{\beta=1}^{p} s_{\alpha \beta}(X) A_{\beta}(Y),
$$

where $s_{\alpha \beta}$ is the normal connection defined by $\nabla_{X}^{\perp} \xi_{\alpha}=\sum_{\beta=1}^{p} s_{\alpha \beta}(X) \xi_{\beta}$. Obviously, $\|\bar{\nabla} B\|^{2}=\sum_{\alpha=1}^{p}\left\|(\bar{\nabla} A)_{\alpha}\right\|^{2}$, thus

$$
\|\bar{\nabla} B\|^{2}=0 \Leftrightarrow\left(\bar{\nabla}_{X} A\right)_{\alpha}=0 \text { for all } \alpha=1, \cdots, p .
$$

The Ricci curvature tensor can be derived from the Gauss equation and the minimality of the focal submanifolds as Ric $=(n-1) \operatorname{Id}-\sum_{\alpha=1}^{p} A_{\alpha}^{2}$. Moreover, by the fact $s_{\alpha \beta}+s_{\beta \alpha}=0$, one has

$$
\nabla_{X} \text { Ric }=-\sum_{\alpha=1}^{p}\left(\nabla_{X} A\right)_{\alpha} A_{\alpha}-\sum_{\alpha=1}^{p} A_{\alpha}\left(\nabla_{X} A\right)_{\alpha}=-\sum_{\alpha=1}^{p}\left(\bar{\nabla}_{X} A\right)_{\alpha} A_{\alpha}-\sum_{\alpha=1}^{p} A_{\alpha}\left(\bar{\nabla}_{X} A\right)_{\alpha},
$$

thus $\|\bar{\nabla} B\|^{2}=0$ implies $\nabla$ Ric $=0$ by (8), namely, Ricci-parallel. Putting all the facts above together, we conclude that

Lemma 3.1. A focal submanifold of an isoparametric hypersurface is semiparallel if and only if it is a parallel submanifold. In particular, a semiparallel focal submanifold must be Ricci-parallel.

\section{Proof of Theorem 1.2.}

(1) For the cases $g=3, n=2 m, p=m+1$, we have

$$
\|B\|^{2}=\frac{2}{3} m(m+1), \quad \sum_{\alpha, \beta=1}^{p}\left\|\left[A_{\alpha}, A_{\beta}\right]\right\|^{2}=\frac{8 m^{2}(m+1)}{9}, \quad\left\langle A_{\alpha}, A_{\beta}\right\rangle=\frac{2 m}{3} \delta_{\alpha \beta} .
$$

Substituting these into Simons formula (2), one has

$$
\begin{aligned}
\|\bar{\nabla} B\|_{M_{ \pm}}^{2} & =\sum_{\alpha, \beta=1}^{p}\left\langle A_{\alpha}, A_{\beta}\right\rangle^{2}+\sum_{\alpha, \beta=1}^{p}\left\|\left[A_{\alpha}, A_{\beta}\right]\right\|^{2}-n\|B\|^{2} \\
& =\frac{\left(4 m^{2}\right)(m+1)}{9}+\frac{8 m^{2}(m+1)}{9}-\frac{4}{3} m^{2}(m+1) \\
& =0,
\end{aligned}
$$

thus all the focal submanifolds with $g=3$ are semiparallel by Lemma 3.1. 
Remark 3.1. Each focal submanifold with $g=3$ must be one of the Veronese embeddings of projective planes $\mathbb{F} P^{2}$ into $\mathbb{S}^{3 m+1}$, where $\mathbb{F}$ is the division algebra $\mathbb{R}, \mathbb{C}$, $\mathbb{H}$, or $\mathbb{O}$ for $m=1,2,4$, or 8 , respectively. In facts, all the standard Veronese embeddings of compact symmetric spaces of rank one into unit spheres are known to be parallel submanifolds $\left[\mathrm{Sak}\right.$. Here we have provided a new proof for the cases of $\mathbb{F} P^{2}$, $\mathbb{F}=\mathbb{R}, \mathbb{C}, \mathbb{H}$ or $\mathbb{O}$.

(2) For the cases $g=4$ and multiplicities $\left(m_{1}, m_{2}\right)$, for the focal submanifolds, say $M_{+}, n=2 m_{2}+m_{1}, p=m_{1}+1$, we have

$$
\|B\|_{M_{+}}^{2}=2 m_{2}\left(m_{1}+1\right), \quad\left\langle A_{\alpha}, A_{\beta}\right\rangle_{M_{+}}=2 m_{2} \delta_{\alpha \beta} .
$$

Recall a formula of Ozeki-Takeuchi (see [OT], p.534, Lemma. 12. (ii))

$$
A_{\alpha}=A_{\beta}^{2} A_{\alpha}+A_{\alpha} A_{\beta}^{2}+A_{\beta} A_{\alpha} A_{\beta}, \quad \alpha \neq \beta .
$$

Multiply by $A_{\alpha}$ and take traces on both sides,

$$
\operatorname{tr}\left(A_{\alpha}^{2}\right)=2 \operatorname{tr}\left(A_{\alpha}^{2} A_{\beta}^{2}\right)+\operatorname{tr}\left(A_{\alpha} A_{\beta}\right)^{2}
$$

then

$$
m_{1}\|B\|^{2}=m_{1}\left(m_{1}+1\right) \operatorname{tr}\left(A_{\alpha}^{2}\right)=2 \sum_{\alpha \neq \beta} \operatorname{tr}\left(A_{\alpha}^{2} A_{\beta}^{2}\right)+\sum_{\alpha \neq \beta} \operatorname{tr}\left(A_{\alpha} A_{\beta}\right)^{2} .
$$

On the other hand, by the formula $\left(A_{\alpha}\right)^{3}=A_{\alpha}$ for $1 \leq \alpha \leq p$ (see [OT] p.534 Lemma. 12. (i)),

$$
3\|B\|^{2}=2 \sum_{\alpha=\beta} \operatorname{tr}\left(A_{\alpha}^{2} A_{\beta}^{2}\right)+\sum_{\alpha=\beta} \operatorname{tr}\left(A_{\alpha} A_{\beta}\right)^{2}
$$

thus

$$
\left(m_{1}+3\right)\|B\|^{2}=2 \sum_{\alpha, \beta=1}^{p} \operatorname{tr}\left(A_{\alpha}^{2} A_{\beta}^{2}\right)+\sum_{\alpha, \beta=1}^{p} \operatorname{tr}\left(A_{\alpha} A_{\beta}\right)^{2} .
$$

Substituting (10) into (44), one concludes that for $M_{+}$

$$
\sum_{\alpha, \beta=1}^{p}\left\|\left[A_{\alpha}, A_{\beta}\right]\right\|_{M_{+}}^{2}=6 \operatorname{tr}\left(\sum_{\alpha=1}^{p} A_{\alpha}^{2} \sum_{\beta=1}^{p} A_{\beta}^{2}\right)-2\left(m_{1}+3\right)\|B\|^{2} .
$$

Similarly, for $M_{-}$,

$$
\sum_{\alpha, \beta=1}^{p}\left\|\left[A_{\alpha}, A_{\beta}\right]\right\|_{M_{-}}^{2}=6 \operatorname{tr}\left(\sum_{\alpha=1}^{p} A_{\alpha}^{2} \sum_{\beta=1}^{p} A_{\beta}^{2}\right)-2\left(m_{2}+3\right)\|B\|^{2} .
$$


Moreover, substituting (9) and (11) into (2), one arrives at

$$
\begin{aligned}
\|\bar{\nabla} B\|_{M_{+}}^{2}= & 4 m_{2}^{2}\left(m_{1}+1\right)+6 \operatorname{tr}\left(\sum_{\alpha=1}^{p} A_{\alpha}^{2} \sum_{\beta=1}^{p} A_{\beta}^{2}\right)-2\left(m_{1}+3\right)\|B\|^{2} \\
& -\left(2 m_{2}+m_{1}\right)\|B\|^{2} \\
= & 6 \operatorname{tr}\left(\sum_{\alpha=1}^{p} A_{\alpha}^{2} \sum_{\beta=1}^{p} A_{\beta}^{2}\right)-6 m_{2}\left(m_{1}+1\right)\left(m_{1}+2\right) .
\end{aligned}
$$

Similarly,

$$
\|\bar{\nabla} B\|_{M_{-}}^{2}=6 \operatorname{tr}\left(\sum_{\alpha=1}^{p} A_{\alpha}^{2} \sum_{\beta=1}^{p} A_{\beta}^{2}\right)-6 m_{1}\left(m_{2}+1\right)\left(m_{2}+2\right) .
$$

We firstly show that the focal submanifolds of the cases $g=4,\left(m_{1}, m_{2}\right)=(7,8)$ or $(8,7)$ can not be semiparallel. In fact, by the inequality $\operatorname{tr}\left(A^{2}\right) \geq \frac{(\operatorname{tr} A)^{2}}{n}$ for symmetric matrices,

$$
\begin{aligned}
\|\bar{\nabla} B\|_{M_{+}}^{2} & \geq \frac{6}{m_{1}+2 m_{2}}\left(\operatorname{tr} \sum_{\alpha=1}^{p} A_{\alpha}^{2}\right)^{2}-6 m_{2}\left(m_{1}+1\right)\left(m_{1}+2\right) \\
& =\frac{24}{m_{1}+2 m_{2}} m_{2}^{2}\left(m_{1}+1\right)^{2}-6 m_{2}\left(m_{1}+1\right)\left(m_{1}+2\right) \\
& =\frac{6 m_{1} m_{2}\left(m_{1}+1\right)}{m_{1}+2 m_{2}}\left(2 m_{2}-m_{1}-2\right),
\end{aligned}
$$

Clearly, in the cases of $\left(m_{1}, m_{2}\right)=(7,8)$ or $(8,7)$, one has $\|\bar{\nabla} B\|_{M_{+}}^{2}>0,\|\bar{\nabla} B\|_{M_{-}}^{2}>0$. By Lemma 3.1. the focal submanifolds of the cases $g=4,\left(m_{1}, m_{2}\right)=(7,8)$ or $(8,7)$ are not semiparallel.

We nextly show the Ricci-parallel focal submanifolds listed in Theorem 1.1 are all semiparallel case by case.

For the $M_{-}$of OT-FKM type with $\left(m_{1}, m_{2}\right)=(1, k)$, which can be characterized as $M_{-}=\left\{(x \cos \theta, x \sin \theta) \mid x \in \mathrm{S}^{k+1}(1), \theta \in \mathrm{S}^{1}(1)\right\}=\mathrm{S}^{1}(1) \times \mathrm{S}^{k+1}(1) /(\theta, x) \sim(\theta+\pi,-x)$ (see [TY1 Proposition 1.1). Clearly, the Ricci tensor of $M_{-}$can be characterized as $\operatorname{Ric}=0 \oplus k \cdot \operatorname{Id}_{(k+1) \times(k+1)}$. However, by the Gauss equation Ric $=(k+1) \operatorname{Id}-\sum_{\alpha=1}^{k+1} \mathrm{~A}_{\alpha}^{2}$, one has

$$
\sum_{\alpha=1}^{k+1} \mathrm{~A}_{\alpha}^{2}=(k+1) \oplus \operatorname{Id}_{(k+1) \times(k+1)} .
$$

Substituting (14) into (13), one arrives at

$$
\|\bar{\nabla} B\|_{M_{-}^{k+2}}^{2}=6\left((k+1)^{2}+(k+1)\right)-6(k+1)(k+2)=0 .
$$

According to Lemma 3.1, $M_{-}$'s of OT-FKM type with $\left(m_{1}, m_{2}\right)=(1, k)$ are semiparallel submanifolds. Furthermore, the isoparametric families of OT-FKM type with 
multiplicities $(2,1),(6,1)$ are congruent to those with multiplicities $(1,2),(1,6)$, respectively $([\underline{\mathrm{FKM}}])$, thus $M_{+}$'s of OT-FKM type with $\left(m_{1}, m_{2}\right)=(2,1)$ or $(6,1)$ are also semiparallel submanifolds.

The $M_{+}$of OT-FKM type with $\left(m_{1}, m_{2}\right)=(4,3)$ (the homogeneous case), as proved in $($ QTY $)$, is an Einstein manifold. In this way,

$$
\sum_{\alpha=1}^{p} \mathrm{~A}_{\alpha}^{2}=\frac{\|B\|^{2}}{10} \cdot \mathrm{Id}_{10 \times 10}=3 \cdot \operatorname{Id}_{10 \times 10} .
$$

Substituting (15) into (12), one arrives at

$$
\|\bar{\nabla} B\|_{M_{+}^{10}}^{2}=6 \cdot 9 \cdot 10-6 \cdot 3 \cdot 5 \cdot 6=0 .
$$

According to Lemma 3.1, $M_{+}$of OT-FKM type with $\left(m_{1}, m_{2}\right)=(4,3)$ (the homogeneous family) is semiparallel submanifold.

The focal submanifold diffeomorphic to $\widetilde{G}_{2}\left(\mathbb{R}^{5}\right)$ in the exceptional homogeneous case with $\left(m_{1}, m_{2}\right)=(2,2)$, as proved in $(\mathrm{QTY})$, is an Einstein manifold. In this way,

$$
\sum_{\alpha=1}^{p} \mathrm{~A}_{\alpha}^{2}=\frac{\|B\|^{2}}{6} \cdot \mathrm{Id}_{6 \times 6}=2 \cdot \operatorname{Id}_{6 \times 6} .
$$

Substituting (16) into (12), one arrives at

$$
\|\bar{\nabla} B\|_{M^{10}}^{2}=6 \cdot 4 \cdot 6-6 \cdot 2 \cdot 3 \cdot 4=0 .
$$

According to Lemma 3.1 , the focal submanifold diffeomorphic to $\widetilde{G}_{2}\left(\mathbb{R}^{5}\right)$ in the exceptional homogeneous case with $\left(m_{1}, m_{2}\right)=(2,2)$ is semiparallel submanifold.

The proof of the second part of Theorem 1.2 is now complete.

(3) For the cases $g=6, n=5 m, p=m+1$, we have

$$
\|B\|^{2}=\frac{20}{3} m(m+1), \quad\left\langle A_{\alpha}, A_{\beta}\right\rangle=\frac{20 m}{3} \delta_{\alpha \beta} .
$$

Substituting these and (3) into Simons formula (2), one has

$$
\begin{aligned}
\|\bar{\nabla} B\|_{M_{+}}^{2} & =\sum_{\alpha, \beta=1}^{p}\left\langle A_{\alpha}, A_{\beta}\right\rangle^{2}+\sum_{\alpha, \beta=1}^{p}\left\|\left[A_{\alpha}, A_{\beta}\right]\right\|^{2}-n\|B\|^{2} \\
& =\frac{\left(400 m^{2}\right)(m+1)}{9}+\left(72+\frac{8}{9}\right) m^{2}(m+1)-\frac{100}{3} m^{2}(m+1) \\
& =84 m^{2}(m+1)>0 .
\end{aligned}
$$

Similarly,

$$
\|\bar{\nabla} B\|_{M_{-}}^{2}=20 m^{2}(m+1)>0 .
$$

Hence, none of the focal submanifolds with $g=6$ are semiparallel by Lemma 3.1.

The proof of Theorem 1.2 is now complete. 
Acknowledgements . The authors are greatly indebted to Professor Zizhou Tang for his valuable suggestions and encouragement.

\section{REFERENCES}

[Abr] U. Abresch, Isoparametric hypersurfaces with four or six distinct principal curvatures, Math. Ann. 264(1983), 283-302.

[Car1] E. Cartan, Familles de surfaces isoparamétriques dans les espaces à courbure constante, Annali di Mat. 17 (1938), 177-191.

[Car2] E. Cartan, Sur des familles remarquables d'hypersurfaces isoparamétriques dans les espaces sphériques, Math. Z. 45 (1939), 335-367.

[CCJ] T. E. Cecil, Q. S. Chi and G. R. Jensen, Isoparametric hypersurfaces with four principal curvatures, Ann. of Math. (2) 166 (2007), no. 1, 1-76.

[Chi] Q. S. Chi, Isoparametric hypersurfaces with four principal curvatures, II and III, Nagoya Math. J. 204 (2011), 1-18, and J. Diff. Geom. 94 (2013), 487-522.

[CdK] S. S. Chern, M. do Carmo and S. Kobayashi, Minimal submanifolds of the sphere with second fundamental form of constant length, in: F. Browder (Ed.), Functional Analysis and Related Fields, Springer-Verlag, Berlin, 1970.

[CR] T. E. Cecil and P. T. Ryan, Tight and taut immersions of manifolds, Research Notes in Math. 107, Pitman, London, 1985.

[Dep] J. Deprez, Semiparallel hypersurfaces, Rend Sem Mat Univ Politec Torino. 44(1986), 303C316.

[DN] J. Dorfmeister and E. Neher, Isoparametric hypersurfaces, case $g=6, m=1$, Communications in Algebra. 13 (1985), 2299-2368.

[FKM] D. Ferus, H. Karcher and H. F. Münzner, Cliffordalgebren und neue isoparametrische Hyperflächen, Math. Z. 177 (1981), 479-502. For an English version, see arXiv: 1112.2780.

[Gra] A. Gray, Einstein like manifolds which are not Einstein, Geom. Ded. 7 (1978), 259-280.

[Imm] S. Immervoll, On the classification of isoparametric hypersurfaces with four distinct principal curvatures in spheres, Ann. of Math. 168 (2008), no. 3, 1011-1024.

[Lum] Ü. Lumiste, Semiparallel Submanifolds in Space Forms, Springer Monographs in Mathematics, Springer, New York, 2009.

[LY] Q. C. Li and W. J. Yan, On Ricci tensor of focal submanifolds of isoparametric hypersurfaces, Science China-Math. 58, 2015, doi: 10.1007/s11425-014-4928-6.

[Miy1] R. Miyaoka, The linear isotropy group of $G_{2} / S O(4)$, the Hopf fibering and isoparametric hypersurfaces, Osaka J. Math. 30 (1993), 179-202.

[Miy2] R. Miyaoka, Geometry of $G_{2}$ orbits and isoparametric hypersurfaces, Nagoya Mathematical Journal 203 (2011), 175-189.

[Miy3] R. Miyaoka, Isoparametric hypersurfaces with $(g, m)=$ (6,2), Ann. Math. 177 (2013), 53-110.

[Mün] H. F. Münzner, Isoparametrische Hyperflächen in Sphären, I and II, Math. Ann. 251 (1980), 57-71 and 256 (1981), 215-232.

[OT] H. Ozeki and M. Takeuchi, On some types of isoparametric hypersurfaces in spheres, I and II, Tôhoku Math. J. 27 (1975), 515-559 and 28 (1976), 7-55.

[QTY] C. Qian, Z. Z. Tang and W. J. Yan, New examples of Willmore submanifolds in the unit sphere via isoparametric functions, II, Ann. Glob. Anal. Geom. 43 (2013), 47-62.

[Sak] K. Sakamoto, Planar geodesic immersions. Tohoku Math. J. (2) 29 (1977), no. 1, 25-56.

[Sim] J. Simons, Minimal varieties in Riemannian manifolds, Ann. Math. 88 (1968) 62 C105. 
[TY1] Z. Z. Tang and W. J. Yan, Isoparametric foliation and Yau conjecture on the first eigenvalue. J. Diff. Geom. 94 (2013), no. 3, 521-540.

[TY2] Z. Z. Tang and W. J. Yan, New examples of Willmore submanifolds in the unit sphere via isoparametric functions, Ann. Glob. Anal. Geom. 42 (2012), 403-410.

[TY3] Z. Z. Tang and W. J. Yan, Isoparametric foliation and a problem of Besse on generalizations of Einstein condition, arXiv:1307.3807, 2013.

[Xie] Y. Q. Xie, Willmore submanifolds in the unit sphere via isoparametric functions, arXiv:1408.0071.

School of Mathematical Sciences, Laboratory of Mathematics and Complex Systems, Beijing Normal University, Beijing 100875, China

E-mail address: qichaoli@mail.bnu.edu.cn

School of Mathematical Sciences, Laboratory of Mathematics and Complex Systems, Beijing Normal University, Beijing 100875, China

E-mail address: zhangli@mail.bnu.edu.cn 